\title{
Full-length transcriptome analysis of Spodoptera frugiperda larval brain reveals detoxification genes
}

\author{
Lei Yang ${ }^{1}$, Binglin Xing ${ }^{1}$, Fen Li ${ }^{1}$, Wang Li kui ${ }^{1}$, Linlin Yuan ${ }^{1}$, Amosi Leonard Mbuji ${ }^{1,2}{ }^{2}$ Peng Zhengqiang ${ }^{3}$, Farag \\ Malhat ${ }^{4}$, Shaoying Wu ${ }^{\text {Corresp. } 1}$ \\ 1 Hainan University, Haikou, Hainan, China \\ 2 Department of Resources utilization and plant protection, College of Resources and Environmental science, China Agricultural University, Beijing, Beijing, \\ China \\ 3 The Ministry of Agriculture and Rural Affairs Key Laboratory of Integrated Pest Management of Tropical Crops, Environment and Plant Protection \\ Institute, Chinese Academy of Tropical Agricultural Sciences, Haikou, Hainan, China \\ 4 Pesticide Residues and Environmental Pollution Department, Agricultural Research Center, Dokki, Giza, Egypt \\ Corresponding Author: Shaoying Wu \\ Email address: wsywsy6000@hainanu.edu.cn
}

Background. Spodoptera frugiperda (J. E. Smith), commonly known as fall armyworm (FAW), is one of the most destructive agricultural pests in the world and has posed a great threat to crops. The improper use of insecticides has led to rapid development of resistance. However, the genetic data available for uncovering the insecticide resistance mechanisms are scarce.

Methods. In this study, we used PacBio single-molecule real-time (SMRT) sequencing aimed at revealing the full-length transcriptome profiling of the FAW larval brain to obtain detoxification genes.

Results. A total of 18,642 high-quality transcripts were obtained with an average length of 2,371 bp, and 11,230 of which were successfully annotated in six public databases. Among these, 5,692 alternative splicing events were identified. 


\section{Full-length transcriptome analysis of Spodoptera}

\section{2 frugiperda larval brain reveals detoxification genes}

3 Lei Yang ${ }^{1}$, Binglin Xing ${ }^{1}$, Fen Li $^{1}$, Likui Wang ${ }^{1}$, Linlin Yuan ${ }^{1}$, Amosi Leonard Mbuji ${ }^{1,2}$,

4 Zhengqiang Peng ${ }^{3}$, Farag Malhat ${ }^{4}$, Shaoying $\mathrm{Wu}^{1}$

$5 \quad{ }^{1}$ Hainan University, Haikou 570228, China

$6 \quad{ }^{2}$ Department of Resources utilization and plant protection, College of Resources and

7 Environmental science, China Agricultural University, Beijing 100193, China

$8{ }^{3}$ The Ministry of Agriculture and Rural Affairs Key Laboratory of Integrated Pest Management

9 of Tropical Crops, Environment and Plant Protection Institute, Chinese Academy of Tropical

10 Agricultural Sciences, Haikou 571101, China

$11{ }^{4}$ Pesticide Residues and Environmental Pollution Department, Central Agricultural Pesticide

12 Laboratory, Agricultural Research Center, Dokki, Giza 12618, Egypt

14 Corresponding Author:

15 Shaoying $\mathrm{Wu}^{1}$

16 Renmin Street 58, Haikou, Hainan, 570228, China

17 Email address: wsywsy6000@hainanu.edu.cn 
19

20

21

22

Abstract

Background. Spodoptera frugiperda (J. E. Smith), commonly known as fall armyworm (FAW), is one of the most destructive agricultural pests in the world and has posed a great threat to crops. The improper use of insecticides has led to rapid development of resistance. However, the genetic data available for uncovering the insecticide resistance mechanisms are scarce.

Methods. In this study, we used PacBio single-molecule real-time (SMRT) sequencing aimed at revealing the full-length transcriptome profiling of the FAW larval brain to obtain detoxification genes.

Results. A total of 18,642 high-quality transcripts were obtained with an average length of 2,371 bp, and 11,230 of which were successfully annotated in six public databases. Among these, 5,692 alternative splicing events were identified. Additionally, five main gene families associated with the detoxification function were classified, mainly including Cytochrome P450s (CYPs), ATP-binding cassettes (ABCs), Carboxylesterases (CESs), Glutathione S-transferases (GSTs) and UDP-glucuronosyl transferases (UGTs). There were 84 CYP transcripts assigned into CYP4, CYP6, CYP9, and CYP12 clades, 54 transcripts of $\mathrm{ABC}$ from five functional groups, 46 transcripts of CES distributed in three families, 33 transcripts of GST distributed in three clades, and 31 UGT transcripts grouped into two families. Notably, these explorative findings will greatly contribute to revealing the resistance mechanisms and further benefit the investigation of gene functions.

\section{Introduction}

Spodoptera frugiperda (J. E. Smith), known as fall armyworm (FAW), belongs to the Noctuidae of Lepidoptera. It is one of the most dangerous invasive pests originated from the tropical and sub-tropical regions of the Americas (Capinera 2002). FAW possesses a wide range of hosts, including at least 353 plants belonging to 76 families, such as corn, wheat, rice, sorghum and others (Wan et al. 2020), it has caused great damages to crops, thus leading to huge economic 
losses to agriculture. FAW is an alien invasive pest in China and quickly invaded the Yunnan Province, southern China in December 2018 (Sun et al. 2021; Wu et al. 2019). At the end of 2020, it had colonized corn fields of 1,338 counties of 27 provinces in China, and the damaged area caused by FAW has reached as high as 11,125-12,780 hectares (Zhou et al. 2021). FAW adult has a strong capability of long-distance migration, and the average flight distance was more than $100 \mathrm{~km}$ per night under proper environmental conditions (Tendeng et al. 2019), which aggravating its rapid spreading (Wan et al. 2020). Additionally, the fecundity of FAW is extremely high, the average number of laying eggs per female is 1,052-1,323 (Wan et al. 2020). More exaggeratedly, the maximum number of egg production per female reached 2,000 in Africa (Prasanna et al. 2018). To sum up, the gluttony, strong migration ability and high fertility of FAW have attracted extensive attention. Therefore, it was classified as the top first destructive pest in China (Zhou et al. 2021).

Transcriptome especially the short-reading sequencing technique has long been developed and used as one of the most efficient methods to obtain reference gene sequences, quantify gene expression levels and characterize gene functions not only in vertebrates, but also in invertebrates such as insects (Djebali et al. 2012; Ekblom \& Galindo 2011; Nagalakshmi et al. 2008; Yin et al. 2014; Yin et al. 2016). Although booming advances in technology have been made recently, there were many limitations (Zhou et al. 2010). For instance, this sequencing technology failed to identify genes longer than 5,000 bp upon most occasions, and the excavation of alternative splicing spanning entire transcripts was rather difficult (Koren et al. 2012). Moreover, incorrect annotations were often conducted due to low-quality transcripts. To overcome these limitations, third generation full-length transcriptome based on PacBio singlemolecule real-time (SMRT) sequencing technology was developed (Picelli et al. 2013). Compared to the short-reading sequencing technique, SMRT sequencing greatly contributes to our understanding of the physiological processes of objects at a molecular level, such as the availability of accurate gene full-length (maximum $>20,000 \mathrm{bp}$ ), identifiable alternative splicing isoforms, and easy to screen novel transcripts. 
Insects are naturally under the selection pressure of various pesticides in local ecological context. As a coping strategy, several defensive mechanisms have been formed to enhance the metabolism of chemicals, decrease their toxic effect and ensure their survival during long-term evolution (Despres et al. 2007). Among these, metabolic resistance is of vital importance, which involves many detoxification enzymes acting in the biotransformation of hazardous chemicals to non-toxic metabolites. To date, many detoxification gene families have been characterized typically classified into five categories according to their different metabolic phases, among which the ATP-binding cassettes (ABCs) are involved in the transport of a broad variety of substrates across the cell membrane (Atsumi et al. 2012), and Carboxylesterases (CESs) hydrolyze an ester via the addition of water to form the corresponding alcohol and acid (Wheelock et al. 2005). In contrast, Cytochrome P450s (CYPs) are terminal oxidases involved in drug and steroid hydroxylation reactions (Scott 1999), and Glutathione S-transferases (GSTs) can catalyze the conjugation of glutathione to insecticides or directly metabolize the insecticides to less toxic soluble conjugate or non-catalytically bind to the insecticides (Kostaropoulos et al. 2001), while UDP-glucuronosyl transferases (UGTs) conjugate the uridine diphosphate glucuronic acid to metabolites to decrease hydrophobicity (Li et al. 2018). The roles of these detoxification genes had been well documented in numerous phytophagous pests such as lepidopteran, coleopteran, hemipteran, dipteran, and other species (Enayati et al. 2005; Stevens et al. 2000; Yang et al. 2005). However, the full-length data of detoxification genes are scarce.

Here, we used PacBio SMRT sequencing technology to profile the full-length transcriptome of the FAW larval brain. Next, five main gene families associated with the detoxification function were classified, and the predicted lncRNA targeting these transcripts were further analyzed. These findings undoubtedly provide important information for exploring the scenario of these genes and revealing their functions.

\section{Materials \& Methods}

\section{Insect rearing and sampling}

FAW larvae were collected from the corn field in Baoting, Hainan, Southern China 
99

100

101

102

103

104

105

106

107

108

109

110

111

112

113

114

115

116

117

118

119

120

121

122

123

124

125

$\left(19^{\circ} 14^{\prime} 45.12^{\prime \prime} \mathrm{N}, 109^{\circ} 89^{\prime} 40.40^{\prime \prime} \mathrm{E}\right)$ on May $5^{\text {th }}, 2019$. We established a laboratory colony by

feeding the larvae on artificial diet under conditions of $25 \pm 1{ }^{\circ} \mathrm{C}, 14 \mathrm{~h}: 10 \mathrm{~h}(\mathrm{~L}: \mathrm{D})$ photoperiod, and $55 \pm 5 \%$ relative humidity. The third instar larvae were individually transferred into a small box and reared until pupation. Adults were transferred into a home-made insect raise cage (Patent no. 201921652702.2) after inclusion and fed with 10\% hydromel (v/v). Once adequate fourth instar larvae were obtained, we rinsed them in $75 \%$ ethanol (v/v) and sterile phosphatebuffered saline (PBS, pH 7.2) twice, respectively. Next, the brain was dissected in PBS containing 1 unit/ $\mu \mathrm{L}$ Murine RNase inhibitor (Vazyme, Nanjing, China) under an Olympus SZ61 stereomicroscope (Olympus, Japan). We collected the sample into a $1.5 \mathrm{ml}$ Eppendorf tube for subsequent RNA extraction.

\section{RNA isolation and SMRT sequencing}

We extracted the total RNA of FAW larval brain using TRIzol reagent (Invitrogen, Carlsbad, United States) according to the description of manufacturer and checked the RNA purity (OD 260/280) using Nanodrop 2000 spectrophotometer (NanoDrop products, USA). Then, RNA degradation and DNA contamination were detected by $1 \%$ agarose gel electrophoresis. After a quality inspection, $12 \mu \mathrm{g}$ RNA was used to gather mRNA molecules by oligo (dT) magnetic beads. We constructed a full length cDNA library using the Clontech SMARTer PCR cDNA synthesis kit (Clontech, CA, USA). The synthesized cDNA was purified by AMPure XP beads (Beckman Coulter, CA, USA) and sequenced on the PacBio sequel II platform (Pacific Biosciences, CA, USA) based on the manufacturer's instructions. Raw data of FAW transcriptome were available from the NCBI SRA database (Project number: PRJNA729608).

\section{Data processing of full-length transcriptome}

We used SMRTlink 6.0 software (Pacific Biosciences, CA, USA) to process the sequence data. Then, reads containing adaptors, Poly-N, and low-quality from raw data were removed, and the filtered reads were converted into cyclic consensus sequence (CCS) according to the criteria of full passes $\geq 1$ and sequence accuracy $>0.9$. Full length and non-full length reads were respectively predicted followed by arrow polishing using Quiver. Subsequently, we removed 
126

127

128

129

130

131

132

133

134

135

136

137

138

139

140

141

142

143

144

145

146

147

148

149

150

151

152

redundant sequences using the CD-hit program and aligned these terms against the FAW reference genome (SRA project number: PRJNA647344) using GMAP (http://researchpub.gene.com/gmap/) (Li \& Godzik 2006). The obtained transcripts were used for the next analysis.

\section{Transcript annotation and alternative splicing analysis}

All transcripts were functionally annotated based on the six databases, NCBI non-redundant protein sequences (NR) database using blastx with e-value $<1 \mathrm{e}^{-5}$ (Pruitt et al. 2007), Protein family (Pfam) database using Hmmscan, a HMMER-based approach for detecting matches to Pfam families (Finn et al. 2011; Finn et al. 2009), a manually annotated and reviewed protein sequence (Swiss-Prot) database (Bairoch \& Apweiler 2000), Clusters of Orthologous Groups of proteins (KOG) database (Tatusov et al. 2000), Kyoto Encyclopedia of Genes and Genomes (KEGG) database using Diamond v0.8.36 software with e-value $<1 \mathrm{e}^{-5}$ (Kanehisa \& Goto 2000), Gene Ontology (GO) database using Blast2GO v2.5 (Conesa et al. 2005; Consortium 2004), individually. To identify alternative splicing events, transcripts were further processed using Astalavista software with default parameters (Foissac \& Sammeth 2007), and we defined alternative splicing events based on the criteria of previous description (Shen et al. 2021).

\section{Identification and phylogeny analysis of detoxification genes}

Five detoxification gene families, CYP, ABC, CES, GST, and UGT, were screened based on the annotation results and further manually verified. A simple modular architecture research tool (SMART) was used for predicting the conserved domains (Letunic \& Bork 2017). We conducted multiple sequence alignments using Clustal Omega (http://www.ebi.ac.uk/Tools/msa/clustalo/) followed by constructing phylogenetic trees based on the maximum likelihood method using Mega-X software with 1,000 bootstrap values (Kumar et al. 2018), which was further edited and visualized using FigTree v1.4.3 software (Institute of Evolutionary Biology, University of Edinburgh, UK).

\section{LncRNA identification, target prediction and motif analysis}

We used four analysis tools, Pfam (Finn et al. 2014), Coding-Non-Coding-Index (CNCI) (Sun et 
153

154

155

156

157

158

159

160

161

162

163

164

165

166

167

168

169

170

171

172

173

174

175

176

177

178

179

al. 2013), Coding Potential Calculator (CPC) (Kong et al. 2007), and Coding Potential Assessment Tool (CPAT) to screen potential lncRNAs (Wang et al. 2013). To ensure the accuracy of these results, transcripts simultaneously recognized by these tools were defined as IncRNAs. In the following, LncTar tool was used to predict the lncRNA-mRNA interactions ( $L i$ et al. 2015), and motif analysis was conducted using the online tool MEME (Bailey et al. 2015).

\section{Results}

\section{Overview of full-length transcriptome data}

The 61.50 GB clean data of fall armyworm (FAW) larval brain full-length transcriptome were obtained by SMRT sequencing. A total of 297,682 circular consensus sequences (CCSs) were generated, and 248,554 sequences containing poly-A tail were identified as full length sequences (Table 1). Among these, 234,863 reads (94.49\%) was classified as non-chimeric sequences with a mean read length of $2371 \mathrm{bp}$. Next, redundant sequences within different transcript clusters were removed using CD-hit program, and 18,642 high quality non-redundant transcripts were identified and used for subsequent analysis.

\section{Transcript annotation}

To reveal the function of 18,642 transcripts, we annotated these terms on six databases. Notably, 9,834, 7,805, 6,821, 6,715, 5,513 and 219 transcripts were annotated in NR, Pfam, Swiss-Prot, GO, KEGG, and KOG databases, respectively. In total, we obtained 11,230 transcripts with valid functional prediction in at least one database. What stood out in Figure 1A was that 7,737 homologous transcripts (68.90\%) best matched the S. litura, and the top seven hits belonged to the Noctuidae of Lepidoptera based on the annotation results in NR database, showing high similarity. Next, all transcripts were annotated in GO database and 6,715 terms were classified into three GO categories, biological process, cellular component, and molecular function (Figure 1B). In biological processes, we found that most of the transcripts were associated with cellular processes followed by metabolic processes and biological regulation. In contrast, the transcripts involved in cell and cell parts were the most abundant terms in the cellular component category. Additionally, in molecular function, the top three annotated transcripts having binding, catalytic 
180

181

182

183

184

185

186

187

188

189

190

191

192

193

194

195

196

197

198

199

200

201

202

203

204

205

206

and transporter activity were respectively identified (Figure 1B). We also sorted these transcripts into different groups according to their participation in KEGG metabolic pathways. Among the top 20 annotated terms, the most enriched categories pertained to adrenergic signaling in the cardiomyocyte pathway (268) followed by the cardiac muscle contraction pathway (254) (Figure 1C). Finally, we annotated 219 transcripts in KOG databases, transcripts participating in "Cytoskeleton", "Energy production and conversion" and "Post-translational modification, protein turnover, chaperones" were the top three abundant terms (Figure 1D).

\section{CYPs}

Based on the functional annotation, 84 transcripts were characterized as CYPs with a mean length of 2,146 bp, further being manually confirmed in NR database. Among these, 64 transcripts with full length were identified. A phylogenetic tree based on the conserved "CYP domain" was constructed, and results showed that these transcripts were distributed in four clades, CYP4, CYP6, CYP9 and CYP12. Among these, CYP9 concluding 28 transcripts made up the largest clade (Fig 2A), indicating their functional redundancy. We further identified a lncRNA targeting the transcript "PB.1240.2", and the predicted binding site was located in the CDS region (Fig 2B). Besides, the conserved motif analysis presented an overview of the conserved amino acid residues among 84 transcripts (Fig 2C).

\section{ABCs}

Fifty-four transcripts encoding ABCs were obtained from the transcriptome of the FAW larval brain, and nine of which did not contain full length. The mean length of 49 ABCs was 4,074 bp. In typical cases, $\mathrm{ABCs}$ were embedded with one or more "ABC transmembrane domain", we thus constructed a phylogenetic tree based on the conserved domains. From the data in Figure $3 \mathrm{~A}$, it was apparent that 54 transcripts clustered into five clades, namely $\mathrm{ABC}-\mathrm{A}, \mathrm{ABC}-\mathrm{B}, \mathrm{ABC}-$ E, ABC-F, and ABC-G. Among these, ABC-A occupied the majority, which contained 18 members. None of the lncRNAs were predicted to bind to the $54 \mathrm{ABC}$ members. Subsequently, several conserved amino acid sites were identified based on the motif analysis (Figure 3B). CESs 
207

208

209

210

211

212

213

214

215

216

217

218

219

220

221

222

223

224

225

226

227

228

229

230

231

232

After removal of redundant sequences, 46 transcripts were annotated as CESs and half of these were equipped with full length with a mean length of 2,493 bp. To classify these transcripts into different categories, a phylogenetic analysis based on the "CES domain" was constructed. What stood out in Figure 4A was that 46 CESs clustered into three branches, namely CES-A, CES-B and CES-C. Again, no predicted lncRNAs targeting these transcripts were disclosed. According to the results of motif analysis, we identified several amino acid residues highly conserved among the CES transcripts (Figure 4B).

\section{GSTs}

According to the functional annotation of transcriptome data in six databases, 33 transcripts were identified as GSTs, in which 18 terms were available of full length and the mean length was 1,781 bp. GST was embedded with at least one "GST domain" in the N-terminal and one "GST domain" in the C-terminal, respectively, we thus assigned these transcripts into different groups based on the evolutionary relationship of GST domains. From the graph below, we could see that three clusters, including "Delta and Epsilon", "Sigma" and "Omega" gathered together, indicating their functional diversity (Figure 5A). The lncRNA target prediction revealed a regulatory lncRNA could bind to the CDS of "PB.3383.1" transcript (Figure 5B). Additionally, the results obtained from motif analysis of 33 transcripts were shown in Figure 5C, and many conserved sites were also revealed.

\section{UGTs}

Thirty-one transcripts containing "UGT domain" were annotated as UGTs and among these 19 full-length sequences were obtained. The mean length of the fully sequenced transcripts was 2,104 bp. Phylogenetic analysis revealed that these transcripts evolved into two branches, "UGT1" and "UGT2" (Figure 6A). Besides, no lncRNAs were identified as the regulator of UGTs. As shown in Figure 6B, there were several conserved amino acid residues identifiable from 31 UGTs.

\section{Discussion}


233 The full transcriptome analysis of FAW larval brain revealed that 18,642 high-quality transcripts, 23411,230 of which were categorized into different functional groups based on homologous blast in 235 six public databases. From the results, we could see that most of the transcripts showed a higher 236 homology with those of S. litura, a closely related species of FAW, which can provide a valuable 237 reference for subsequent gene function characterization of FAW. Moreover, GO enrichment 238 analysis, KEGG metabolic pathway, and KOG annotations also provided us a reference for the 239 function of these transcripts. Alternative splicing events form diverse gene variants, and 5,692 240 alternative splicing events were identified in this assay, possibly accounting for the 241 diversification of gene functions of these transcripts.

242 The next section of the survey was concerned with detoxification genes. An abundance of 243 evidence has accumulated over the last few decades, indicating that insect herbivores have evolved a set of detoxification genes to detoxify chemicals (Rane et al. 2019). In typical cases, the detoxification enzymes include CYPs, ABCs, CESs, GSTs, UGTs and so on (Georghiou 2012). As one of the largest superfamily, CYPs commonly exist in insects while the number varies a lot. For instance, there was a total of 143 genes in Tribolium castaneum (Zhu et al. 2013). Comparatively, only 38 CYPs were identified in Pediculus humanus humanus (Lee et al. 2010). A previous study has examined CYPs of FAW by whole genome-wide sequencing and finally identified 152 CYP gene clusters (Gui et al. 2020). These results also corroborated the ideas of Xiao et al. (2020), who discovered 169 FAW CYPs with widespread expression at different developmental stages (Xiao et al. 2020). In this study, only 84 transcripts encoding CYPs were annotated in full-length transcriptome data of the FAW larval brain, showing that numerous CYPs were not specifically expressed in the brain. Phylogenetic analysis clustered these transcripts into four clans, among these 15 unique terms were classified into the CYP6 clade. It has been widely known that CYP6 family members act in detoxifying pesticides (Ibrahim et al. 2016; Shi et al. 2018; Yang et al. 2020). Therefore, our findings will provide an 258 important reference for exploring the molecular mechanism of FAW CYPs in detoxification. The 
259

260

261

262

263

264

265

266

267

268

269

270

271

272

273

274

275

276

277

278

279

280

281

282

283

284

285

motif analysis displayed several conserved amino acid sites among 84 CYPs possibly crucial for its function.

Relative to CYPs, ABCs are a set of newly identified detoxification enzymes, among which the ABC-C2 genes are largely responsible for transport of activated Cry toxins into the cell, and the insecticide resistance is derived from loss of function in ABC-C2 (Merzendorfer 2014). Recently, the whole genome sequencing of various insect species provides a great convenience to identify the $\mathrm{ABC}$ gene family. For example, $104,73,56$ and $53 \mathrm{ABCs}$ were characterized in Tetranychus urticae (Dermauw et al. 2013), T. castaneum (Broehan et al. 2013), Drosophila melanogaster (Liu et al. 2011), and Bombyx mori (Liu et al. 2011), respectively. A recent study revealed that $57 \mathrm{ABCs}$ were expressed in FAW combining genome and transcriptome analyses (Gui et al. 2020). In this assay, 54 transcripts were identified, which was in accordance with the previous results. These $\mathrm{ABCs}$ were categorized into five functional groups, $\mathrm{ABC}-\mathrm{A}, \mathrm{ABC}-\mathrm{B}$, $\mathrm{ABC}-\mathrm{E}, \mathrm{ABC}-\mathrm{F}$ and $\mathrm{ABC}-\mathrm{G}$. Several evidences have shown that $\mathrm{ABCs}$ are closely related to insecticide resistance in insects. For instance, in Trichoplusia ni, a significant increase in transcript levels in ABC-B genes was recorded after exposure to deltamethrin (Simmons et al. 2013). Besides, five ABC-B and 13 ABC-C genes of Anopheles gambiae were thought to be responsible for detoxification (Roth et al. 2003). Many conserved amino acid residues were also identified among $54 \mathrm{ABCs}$ by motif analysis, implying the potential targets for developing novel pesticides.

As a member of the large carboxylesterase family, CESs are also implicated in the detoxification of various xenobiotic compounds, especially the organophosphate insecticides (Wheelock et al. 2005). CESs have been well characterized in many flies and aphids, such as Lucilia cuprina (Jackson et al. 2013), D. melanogaster (Cui et al. 2015), Sitobion avenae (Xu et al. 2014), Aphis gossypii (Cao et al. 2008), Myzus persicae (Devonshire et al. 1986). Many studies supported that CESs act in alleviating insecticidal efficacy through gene amplification or up-regulated their transcriptional level (Hemingway 2000). Here, we annotated 46 FAW CESs embedded with many conserved amino acid residues, more than the 30 terms previously reported 
286

287

288

289

290

291

292

293

294

295

296

297

298

299

300

301

302

303

304

305

306

307

308

309

310

311

312

(Gui et al. 2020), and then assigned these terms into three clades from an evolutionary

perspective. We thus speculated that these CESs may account for the rapid development of FAW resistance to pesticides.

GSTs can produce resistance to all main classes of insecticides. Advances in genomic and transcriptomic sequencing technologies triggered an exhaustive identification of GSTs in many insects, including 11 crop pests and four species of mosquito (Pavlidi et al. 2018). Our present investigation identified 33 GSTs distributed in three clades equipped with many conserved amino acid residues. In contrast, a recent study reported 29 GSTs of FAW combining genomic and transcriptomic analyses (Gui et al. 2020). Among these, 15 genes were remarkably downregulated after pesticide treatment. Also, in S. litura, a closely related species of FAW, 37 transcripts were annotated and their expression levels after chlorpyrifos treatment were analyzed (Zhang et al. 2016). These findings undoubtedly provided important references for further functional study of GSTs in detoxification.

UGTs also belong to the relatively newly identified classes. As a kind of detoxification enzyme, UGTs widely exist in lepidopteran insects, including B. mori (Huang et al. 2008), S. litura and many other species (Shen et al. 2021; Shi et al. 2019). In typical cases, UGTs are classified into two distinct subfamilies, UGT1 and UGT2. Compared with the 49 UGTs previously detected in FAW genome and transcriptome (Gui et al. 2020), a small number of UGTs representing 31 transcripts grouped into two families were annotated in this study. It was noted that 17 FAW UGTs showed significant differential expression after pesticide treatment (Gui et al. 2020). Similarly, 10 UGTs were remarkably up-regulated in both indoor and field indoxacarb-resistant strains of S. litura (Shi et al. 2019). Taken all these findings together, we speculated that the 31 UGT transcripts aid in the detoxification process. We also identified several conserved amino acid residues in these UGTs, providing promising targets for developing novel pesticides.

LncRNAs are implicated in various biological functions such as protein synthesis, RNA transport and etc., one of the most important roles in which is the gene expression regulation 
313 through gene silencing (Wang \& Chang 2011). The full transcriptome sequencing provides an 314 alternative method to predict lncRNAs with the potential binding ability to transcripts. Thus, we 315 analyzed the lncRNAs targeting the transcripts of five main detoxification gene families, and the 316 results, while preliminary, suggested that only two lncRNAs potentially bond to the transcript 317 PB.1240.2, a member of CYPs, and the transcript PB.3383.1, a member of GSTs, respectively. 318 These findings may help us understand the underlying molecular mechanism that lncRNAs 319 regulate detoxification genes.

320

321

322

323

\section{Conclusions}

Full-length transcriptome of the FAW larval brain was obtained based on the PacBio SMRT sequencing and analyses involving gene annotation, lncRNA prediction and alternative splicing events were conducted. A further in-depth study examined the five detoxification gene families, which provided an abundant genetic resource and laid a solid foundation for revealing the resistance mechanisms of FAW to pesticides.

\section{References}

Atsumi S, Miyamoto K, Yamamoto K, Narukawa J, Kawai S, Sezutsu H, Kobayashi I, Uchino K, Tamura T, and Mita K. 2012. Single amino acid mutation in an ATP-binding cassette transporter gene causes resistance to Bt toxin Cry1 Ab in the silkworm, Bombyx mori. Proceedings of the National Academy of Sciences of the United States of America 109:E1591-E1598.

Bailey TL, Johnson J, Grant CE, and Noble WS. 2015. The MEME suite. Nucleic Acids Research 43:W39W49.

Bairoch A, and Apweiler R. 2000. The SWISS-PROT protein sequence database and its supplement TrEMBL in 2000. Nucleic Acids Research 28:45-48.

Broehan G, Kroeger T, Lorenzen M, and Merzendorfer H. 2013. Functional analysis of the ATP-binding cassette (ABC) transporter gene family of Tribolium castaneum. BMC Genomics 14:1-19.

Cao CW, Zhang J, Gao XW, Liang P, and Guo HL. 2008. Overexpression of carboxylesterase gene associated with organophosphorous insecticide resistance in cotton aphids, Aphis gossypii (Glover). Pesticide Biochemistry and Physiology 90:175-180.

Capinera JL. 2002. Fall Armyworm, Spodoptera frugiperda (J.E. Smith) (Insecta: Lepidoptera: Noctuidae). University of Florida 2002:1-7.

Conesa A, Götz S, García-Gómez JM, Terol J, Talón M, and Robles M. 2005. Blast2GO: a universal tool for annotation, visualization and analysis in functional genomics research. Bioinformatics 21:3674- 
344

3676.

Consortium GO. 2004. The Gene Ontology (GO) database and informatics resource. Nucleic Acids Research 32:D258-D261.

Cui F, Li MX, Chang HJ, Mao Y, Zhang HY, Lu L-X, Yan SG, Lang ML, Liu L, and Qiao CL. 2015. Carboxylesterase-mediated insecticide resistance: Quantitative increase induces broader metabolic resistance than qualitative change. Pesticide Biochemistry and Physiology 121:88-96.

Dermauw W, Osborne EJ, Clark RM, Grbić M, Tirry L, and Van Leeuwen T. 2013. A burst of ABC genes in the genome of the polyphagous spider mite Tetranychus urticae. BMC Genomics 14:1-22.

Despres L, David JP, and Gallet C. 2007. The evolutionary ecology of insect resistance to plant chemicals. Trends in Ecology \& Evolution 22:298-307. 10.1016/j.tree.2007.02.010

Devonshire A, Moores G, and Ffrench-Constant R. 1986. Detection of insecticide resistance by immunological estimation of carboxylesterase activity in Myzus persicae (Sulzer) and cross reaction of the antiserum with Phorodon humuli (Schrank)(Hemiptera: Aphididae). Bulletin of Entomological Research 76:97-107.

Djebali S, Davis CA, Merkel A, Dobin A, Lassmann T, Mortazavi A, Tanzer A, Lagarde J, Lin W, and Schlesinger F. 2012. Landscape of transcription in human cells. Nature 489:101-108.

Ekblom R, and Galindo J. 2011. Applications of next generation sequencing in molecular ecology of nonmodel organisms. Heredity 107:1-15.

Enayati AA, Ranson H, and Hemingway J. 2005. Insect glutathione transferases and insecticide resistance. Insect Molecular Biology 14:3-8.

Finn RD, Bateman A, Clements J, Coggill P, Eberhardt RY, Eddy SR, Heger A, Hetherington K, Holm L, and Mistry J. 2014. Pfam: the protein families database. Nucleic Acids Research 42:D222-D230.

Finn RD, Clements J, and Eddy SR. 2011. HMMER web server: interactive sequence similarity searching. Nucleic Acids Research 39:W29-W37.

Finn RD, Mistry J, Tate J, Coggill P, Heger A, Pollington JE, Gavin OL, Gunasekaran P, Ceric G, Forslund K, Holm L, Sonnhammer ELL, Eddy SR, and Bateman A. 2009. The Pfam protein families database. Nucleic Acids Research 38:D211-D222. 10.1093/nar/gkp985

Foissac S, and Sammeth M. 2007. ASTALAVISTA: dynamic and flexible analysis of alternative splicing events in custom gene datasets. Nucleic Acids Research 35:W297-W299.

Georghiou GP. 2012. Pest resistance to pesticides: Springer Science \& Business Media.

Gui F, Lan T, Zhao Y, Guo W, Dong Y, Fang D, Liu H, Li H, Wang H, Hao R, Cheng X, Li Y, Yang P, Sahu SK, Chen Y, Cheng L, He S, Liu P, Fan G, Lu H, Hu G, Dong W, Chen B, Jiang Y, Zhang Y, Xu H, Lin F, Slipper B, Postma A, Jackson M, Abate BA, Tesfaye K, Demie AL, Bayeleygne MD, Degefu DT, Chen F, Kuria PK, Kinyua ZM, Liu TX, Yang H, Huang F, Liu X, Sheng J, and Kang L. 2020. Genomic and transcriptomic analysis unveils population evolution and development of pesticide resistance in fall armyworm Spodoptera frugiperda. Protein \& cell. 10.1007/s13238-020- 
380

381

382

383

384

385

386

387

388

389

390

391

392

393

394

395

396

397

398

399

400

401

402

403

404

405

406

407

408

409

410

411

412

413

414

415

00795-7

Hemingway J. 2000. The molecular basis of two contrasting metabolic mechanisms of insecticide resistance. Insect Biochemistry and Molecular Biology 30:1009-1015.

Huang FF, Chai CL, Zhang Z, Liu ZH, Dai FY, Lu C, and Xiang ZH. 2008. The UDP-glucosyltransferase multigene family in Bombyx mori. BMC Genomics 9:563. 10.1186/1471-2164-9-563

Ibrahim SS, Riveron JM, Stott R, Irving H, and Wondji CS. 2016. The cytochrome P450 CYP6P4 is responsible for the high pyrethroid resistance in knockdown resistance-free Anopheles arabiensis. Insect Biochemistry and Molecular Biology 68:23-32.

Jackson CJ, Liu JW, Carr PD, Younus F, Coppin C, Meirelles T, Lethier M, Pandey G, Ollis DL, and Russell RJ. 2013. Structure and function of an insect $\alpha$-carboxylesterase ( $\alpha$ Esterase7) associated with insecticide resistance. Proceedings of the National Academy of Sciences of the United States of America 110:10177-10182.

Kanehisa M, and Goto S. 2000. KEGG: kyoto encyclopedia of genes and genomes. Nucleic Acids Research 28:27-30.

Kong L, Zhang Y, Ye ZQ, Liu XQ, Zhao SQ, Wei L, and Gao G. 2007. CPC: assess the protein-coding potential of transcripts using sequence features and support vector machine. Nucleic Acids Research 35:W345-W349.

Koren S, Schatz MC, Walenz BP, Martin J, Howard JT, Ganapathy G, Wang Z, Rasko DA, McCombie WR, and Jarvis ED. 2012. Hybrid error correction and de novo assembly of single-molecule sequencing reads. Nature Biotechnology 30:693-700.

Kostaropoulos I, Papadopoulos AI, Metaxakis A, Boukouvala E, and Papadopoulou-Mourkidou E. 2001. Glutathione S-transferase in the defence against pyrethroids in insects. Insect Biochemistry and Molecular Biology 31:313-319.

Kumar S, Stecher G, Li M, Knyaz C, and Tamura K. 2018. MEGA X: molecular evolutionary genetics analysis across computing platforms. Molecular Biology and Evolution 35:1547-1549. 10.1093/molbev/msy096

Lee SH, Kang JS, Min JS, Yoon KS, Strycharz JP, Johnson R, Mittapalli O, Margam VM, Sun W, and Li HM. 2010. Decreased detoxification genes and genome size make the human body louse an efficient model to study xenobiotic metabolism. Insect Molecular Biology 19:599-615.

Letunic I, and Bork P. 2017. 20 years of the SMART protein domain annotation resource. Nucleic Acids Research 46:D493-D496. 10.1093/nar/gkx922

Li J, Ma W, Zeng P, Wang J, Geng B, Yang J, and Cui Q. 2015. LncTar: a tool for predicting the RNA targets of long noncoding RNAs. Briefings in Bioinformatics 16:806-812.

Li W, and Godzik A. 2006. Cd-hit: a fast program for clustering and comparing large sets of protein or nucleotide sequences. Bioinformatics 22:1658-1659.

Li X, Shi H, Gao X, and Liang P. 2018. Characterization of UDP-glucuronosyltransferase genes and their

Peer] reviewing PDF | (2021:05:61034:2:0:NEW 21 Jul 2021) 
416

417

418

419

420

421

422

423

424

425

426

427

428

429

430

431

432

433

434

435

436

437

438

439

440

441

442

possible roles in multi-insecticide resistance in Plutella xylostella (L.). Pest Management Science 74:695-704.

Liu S, Zhou S, Tian L, Guo E, Luan Y, Zhang J, and Li S. 2011. Genome-wide identification and characterization of ATP-binding cassette transporters in the silkworm, Bombyx mori. BMC Genomics 12:1-15.

Merzendorfer H. 2014. $A B C$ transporters and their role in protecting insects from pesticides and their metabolites. Advances in Insect Physiology 46:1-72.

Nagalakshmi U, Wang Z, Waern K, Shou C, Raha D, Gerstein M, and Snyder M. 2008. The transcriptional landscape of the yeast genome defined by RNA sequencing. Science 320:1344-1349.

Pavlidi N, Vontas J, and Van Leeuwen T. 2018. The role of glutathione S-transferases (GSTs) in insecticide resistance in crop pests and disease vectors. Current Opinion in Insect Science 27:97-102.

Picelli S, Björklund ÅK, Faridani OR, Sagasser S, Winberg G, and Sandberg R. 2013. Smart-seq2 for sensitive full-length transcriptome profiling in single cells. Nature Methods 10:1096-1098.

Prasanna B, Huesing J, Eddy R, and Peschke V. 2018. Fall armyworm in Africa: a guide for integrated pest management. Wallingford: CAB International, 1-109.

Pruitt KD, Tatusova T, and Maglott DR. 2007. NCBI reference sequences (RefSeq): a curated non-redundant sequence database of genomes, transcripts and proteins. Nucleic Acids Research 35:D61-D65.

Rane RV, Ghodke AB, Hoffmann AA, Edwards OR, Walsh TK, and Oakeshott JG. 2019. Detoxifying enzyme complements and host use phenotypes in 160 insect species. Current Opinion in Insect Science 31:131-138. 10.1016/j.cois.2018.12.008

Roth CW, Holm I, Graille M, Dehoux P, Rzhetsky A, Wincker P, Weissenbach J, and Brey PT. 2003. Identification of the Anopheles gambiae ATP-binding cassette transporter superfamily genes. Molecules and Cells 15:150-158.

Scott JG. 1999. Cytochromes $\mathrm{P} 450$ and insecticide resistance. Insect Biochemistry and Molecular Biology 29:757-777.

Shen GM, Ou SY, He C, Liu J, and He L. 2021. Full length sequencing reveals novel transcripts of detoxification genes along with related alternative splicing events and lncRNAs in Phyllotreta striolata. PloS One 16:e0248749. 10.1371/journal.pone.0248749

Shi L, Shi Y, Zhang Y, and Liao X. 2019. A systemic study of indoxacarb resistance in Spodoptera litura revealed complex expression profiles and regulatory mechanism. Scientific Reports 9:14997. 10.1038/s41598-019-51234-5

Shi Y, Wang H, Liu Z, Wu S, Yang Y, Feyereisen R, Heckel DG, and Wu Y. 2018. Phylogenetic and functional characterization of ten P450 genes from the CYP6AE subfamily of Helicoverpa armigera involved in xenobiotic metabolism. Insect Biochemistry and Molecular Biology 93:79-91.

Simmons J, D'Souza O, Rheault M, and Donly C. 2013. Multidrug resistance protein gene expression in Trichoplusia ni caterpillars. Insect Molecular Biology 22:62-71. 
Stevens JL, Snyder MJ, Koener JF, and Feyereisen R. 2000. Inducible P450s of the CYP9 family from larval Manduca sexta midgut. Insect Biochemistry and Molecular Biology 30:559-568.

Sun L, Luo H, Bu D, Zhao G, Yu K, Zhang C, Liu Y, Chen R, and Zhao Y. 2013. Utilizing sequence intrinsic composition to classify protein-coding and long non-coding transcripts. Nucleic Acids Research 41:e166-e166.

Sun XX, Hu CX, Jia HR, Wu QL, Shen XJ, Zhao SY, Jiang YY, and Wu KM. 2021. Case study on the first immigration of fall armyworm, Spodoptera frugiperda invading into China. Journal of Integrative Agriculture 20:664-672.

Tatusov RL, Galperin MY, Natale DA, and Koonin EV. 2000. The COG database: a tool for genome-scale analysis of protein functions and evolution. Nucleic Acids Research 28:33-36.

Tendeng E, Labou B, Diatte M, Djiba S, and Diarra K. 2019. The fall armyworm Spodoptera frugiperda (J.E. Smith), a new pest of maize in Africa: biology and first native natural enemies detected. International Journal of Biological and Chemical Sciences 13. 10.4314/ijbcs.v13i2.35

Wan J, Huang C, Li Cy, Zhou Hx, Ren Yl, Li Zy, Xing Ls, Zhang B, Qiao X, Liu B, Liu Ch, Xi Y, Liu Wx, Wang Wk, Qian Wq, Mckirdy S, and Wan Fh. 2020. Biology, invasion and management of the agricultural invader: Fall armyworm, Spodoptera frugiperda (Lepidoptera: Noctuidae). Journal of Integrative Agriculture 19:2-19. 10.1016/S2095-3119(20)63367-6

Wang KC, and Chang HY. 2011. Molecular mechanisms of long noncoding RNAs. Molecular Cell 43:904914. 10.1016/j.molcel.2011.08.018

Wang L, Park HJ, Dasari S, Wang S, Kocher J-P, and Li W. 2013. CPAT: Coding-Potential Assessment Tool using an alignment-free logistic regression model. Nucleic Acids Research 41:e74-e74.

Wheelock CE, Shan G, and Ottea J. 2005. Overview of carboxylesterases and their role in the metabolism of insecticides. Journal of Pesticide Science 30:75-83.

Wu Q, Jiang Y, and Wu K. 2019. Analysis of migration routes of the fall armyworm Spodoptera frugiperda (JE Smith) from Myanmar to China. Plant Protection 45:1-6.

Xiao H, Ye X, Xu H, Mei Y, Yang Y, Chen X, Yang Y, Liu T, Yu Y, and Yang W. 2020. The genetic adaptations of fall armyworm Spodoptera frugiperda facilitated its rapid global dispersal and invasion. Molecular Ecology Resources 20:1050-1068.

Xu L, Duan X, Lv Y, Zhang X, Nie Z, Xie C, Ni Z, and Liang R. 2014. Silencing of an aphid carboxylesterase gene by use of plant-mediated RNAi impairs Sitobion avenae tolerance of Phoxim insecticides. Transgenic Research 23:389-396.

Yang X, Deng S, Wei X, Yang J, Zhao Q, Yin C, Du T, Guo Z, Xia J, and Yang Z. 2020. MAPK-directed activation of the whitefly transcription factor CREB leads to P450-mediated imidacloprid resistance. Proceedings of the National Academy of Sciences of the United States of America 117:10246-10253.

Yang Z, Zhang F, He Q, and He G. 2005. Molecular dynamics of detoxification and toxin-tolerance genes in brown planthopper (Nilaparvata lugens Stål., Homoptera: Delphacidae) feeding on resistant rice plants.

Peer] reviewing PDF | (2021:05:61034:2:0:NEW 21 Jul 2021) 
488

489

490

491

492

493

494

495

496

497

498

499

500

501

502

503

Archives of Insect Biochemistry and Physiology 59:59-66.

Yin C, Liu Y, Liu J, Xiao H, Huang S, Lin Y, Han Z, and Li F. 2014. ChiloDB: a genomic and transcriptome database for an important rice insect pest Chilo suppressalis. Database 2014:1-7.

Yin C, Shen G, Guo D, Wang S, Ma X, Xiao H, Liu J, Zhang Z, Liu Y, and Zhang Y. 2016. InsectBase: a resource for insect genomes and transcriptomes. Nucleic Acids Research 44:D801-D807.

Zhang N, Liu J, Chen S-N, Huang L-H, Feng Q-L, and Zheng S-C. 2016. Expression profiles of glutathione S-transferase superfamily in Spodoptera litura tolerated to sublethal doses of chlorpyrifos. Insect Science 23:675-687. 10.1111/1744-7917.12202

Zhou X, Ren L, Meng Q, Li Y, Yu Y, and Yu J. 2010. The next-generation sequencing technology and application. Protein \& cell 1:520-536.

Zhou Y, Wu Ql, Zhang Hw, and Wu Km. 2021. Spread of invasive migratory pest Spodoptera frugiperda and management practices throughout China. Journal of Integrative Agriculture 20:2-10. 10.1016/S20953119(21)63621-3

Zhu F, Moural TW, Shah K, and Palli SR. 2013. Integrated analysis of cytochrome P450 gene superfamily in the red flour beetle, Tribolium castaneum. BMC Genomics 14:1-12. 


\section{Table $\mathbf{1}$ (on next page)}

Table 1: Overview of FAW larval brain full-length transcriptome 
Table 1: Overview of FAW larval brain full-length transcriptome

\begin{tabular}{cc}
\hline Category & Number \\
\hline Circular consensus sequences (CCS) & 297,682 \\
Full-length sequences & 248,554 \\
Non-chimeric full-length sequences with Poly-A Tail & 234,863 \\
Average read length & 2,371 \\
High quality non-redundant transcripts & 18,642 \\
Alternative splicing events & 5,692 \\
Predicted lncRNA & 1,319 \\
Transcripts with functional annontation & 11,230 \\
Transcripts with ORF & 11,013 \\
\hline
\end{tabular}

1 
Figure 1

Figure 1: Full-length transcript annotation of FAW larval brain.

(A) Homologous species distribution of transcripts in NR database. (B) GO functional

categorization of transcripts. (C) Top 20 KEGG functional classifications of transcripts. (D)

COG functional classification of transcripts.

A.

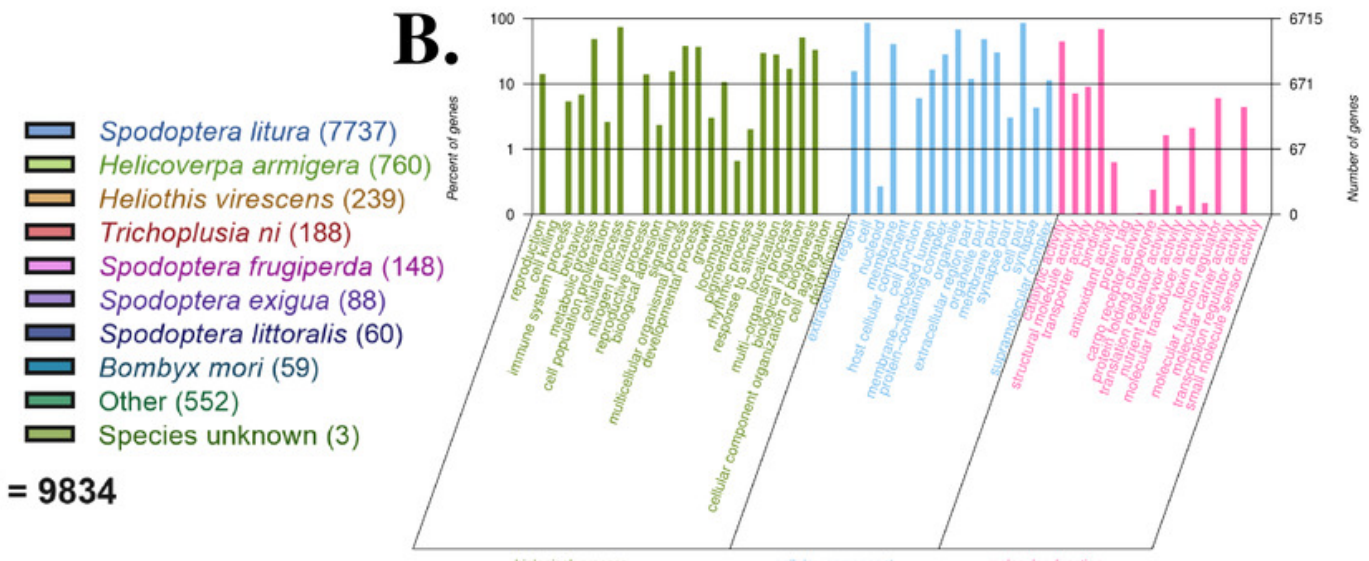

C.
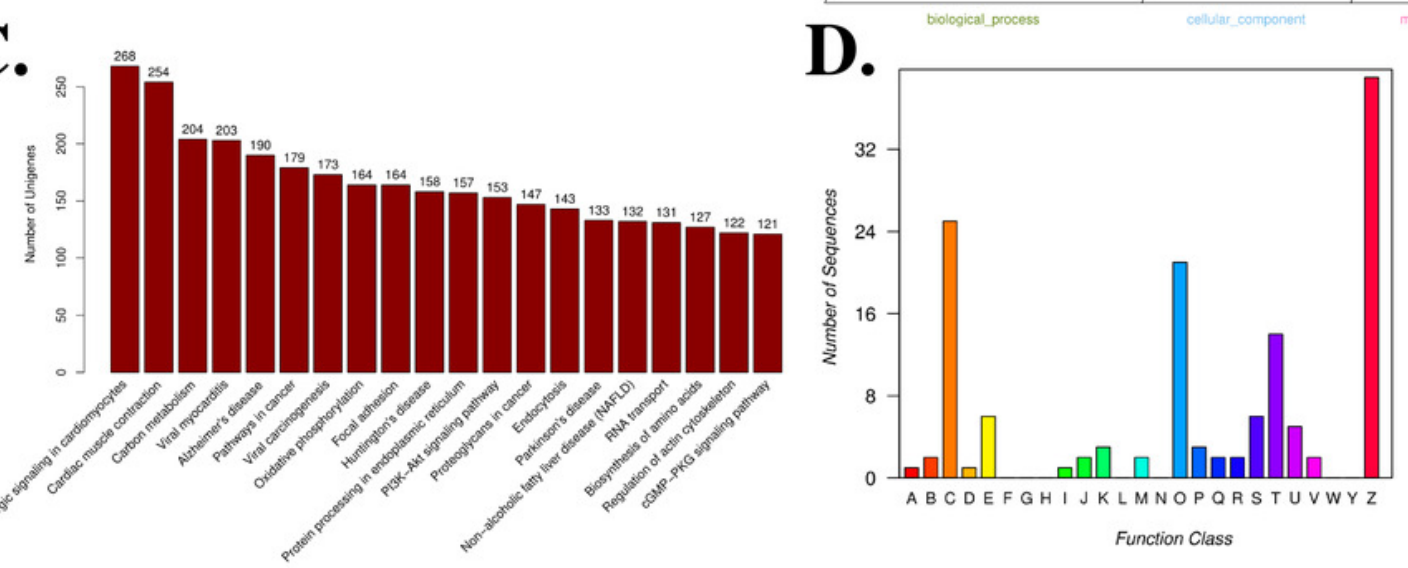

A: ANu procosssong ard mostcastion

8: Cremath arvetuve and onmemics

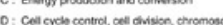

E: Amino asd transpot tand motabolient

F: Nuclosobide transport and motabolism

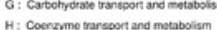

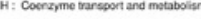

1: Lis transport and metasolicam

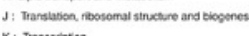

k: Transolpecon

L: Resictionon, necombination and repar

a) Car walim

N. Cort retion

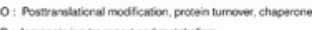

: :

$R$ : Generas tuneten preceston ony

s: Fuction unverom

T: Signa transoustion mecharisms

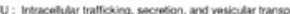

Function Class

V: Detenste mactaniars

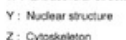


Figure 2

Figure 2: Phylogenetic construction, IncRNA target prediction and motif analysis of CYPs.

(A) Phylogenetic tree of 84 FAW CYP transcripts. The evolutionary tree was constructed based on the maximum likelihood method using the program Mega $X$ with 1000 bootstrap values. Forty-eight different amino acid substitution models were tested and "LG+G" was the best model. (B) The binding sites of IncRNA targeting transcript "PB.1240.2". LncTar was used to predict RNA targets of IncRNAs with a cutoff of -0.1 normalized deltaG. (C) Motif analysis of 84 CYPs. We used MEME tool to identify the conserved motifs.

A.

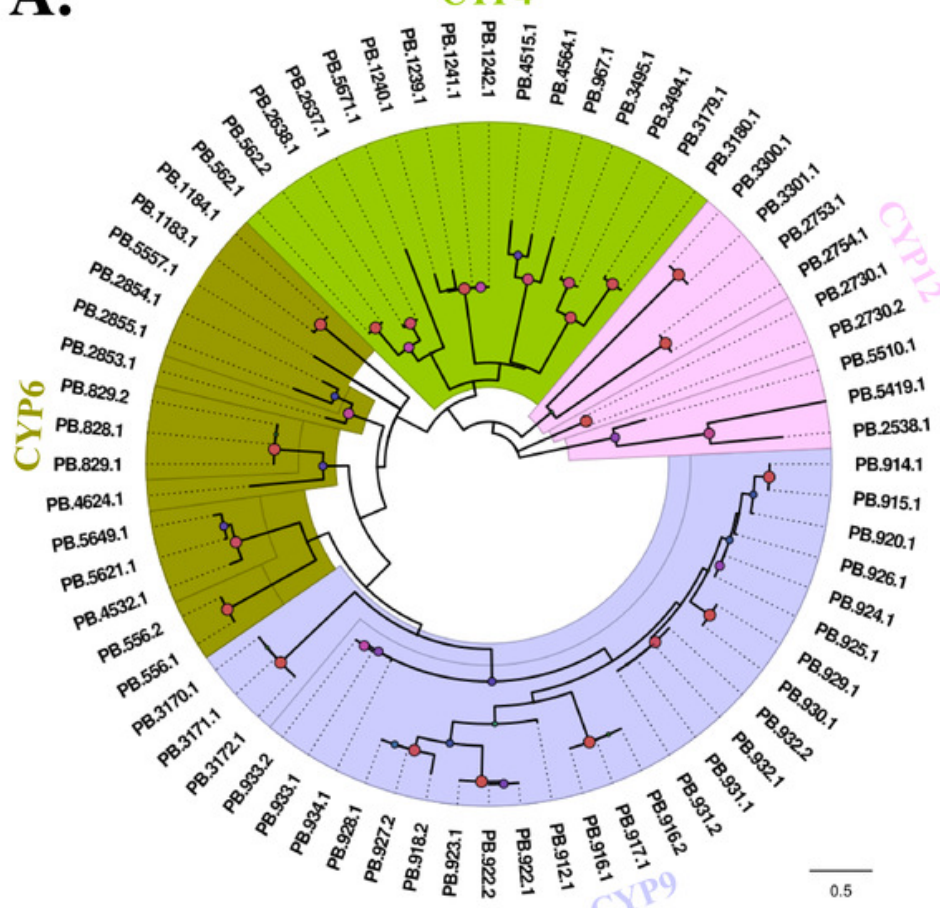

B.

Target: PB.1240.2 5'-AGTCGCTCAGGATCCAGAGTTGAGTGCTGA-3' \|\|$|\||||||||$ LncRNA: transcipt 3'-GTTAAGGATCCT GGTCGCGACTTGCGATT-5'
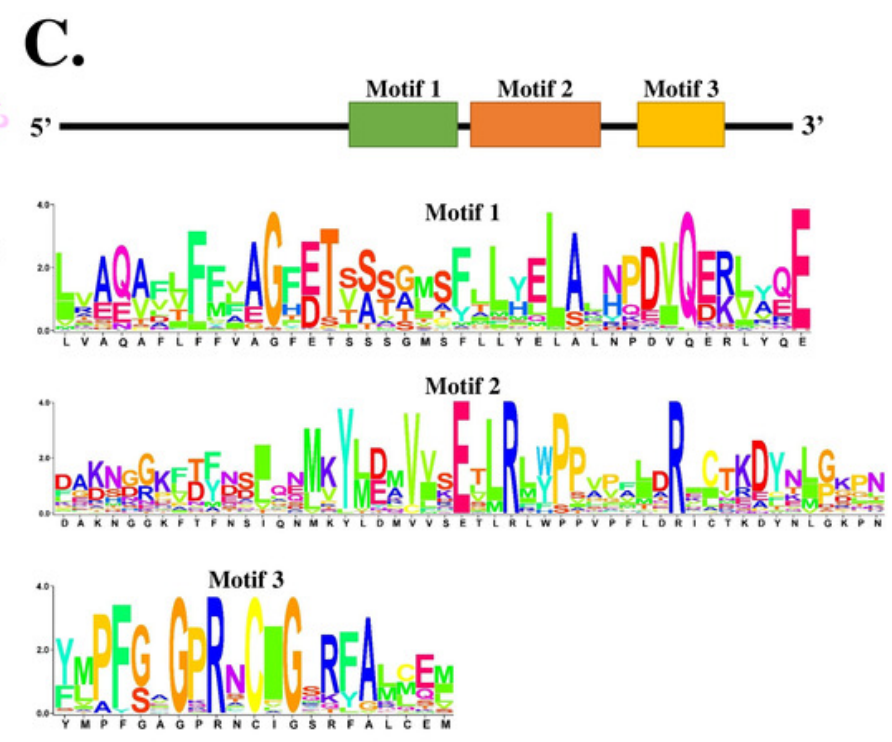
Figure 3

Figure 3: Phylogenetic and motif analysis of ABCs.

(A) Phylogenetic tree of 54 FAW ABC transcripts. The evolutionary tree was constructed based on the maximum likelihood method using the program Mega $\mathrm{X}$ with 1000 bootstrap values. Forty-eight different amino acid substitution models were tested and " $\mathrm{GG}+\mathrm{G}$ " was the best model. (B) Motif analysis of 54 ABCs. We used MEME tool to identify the conserved motifs.
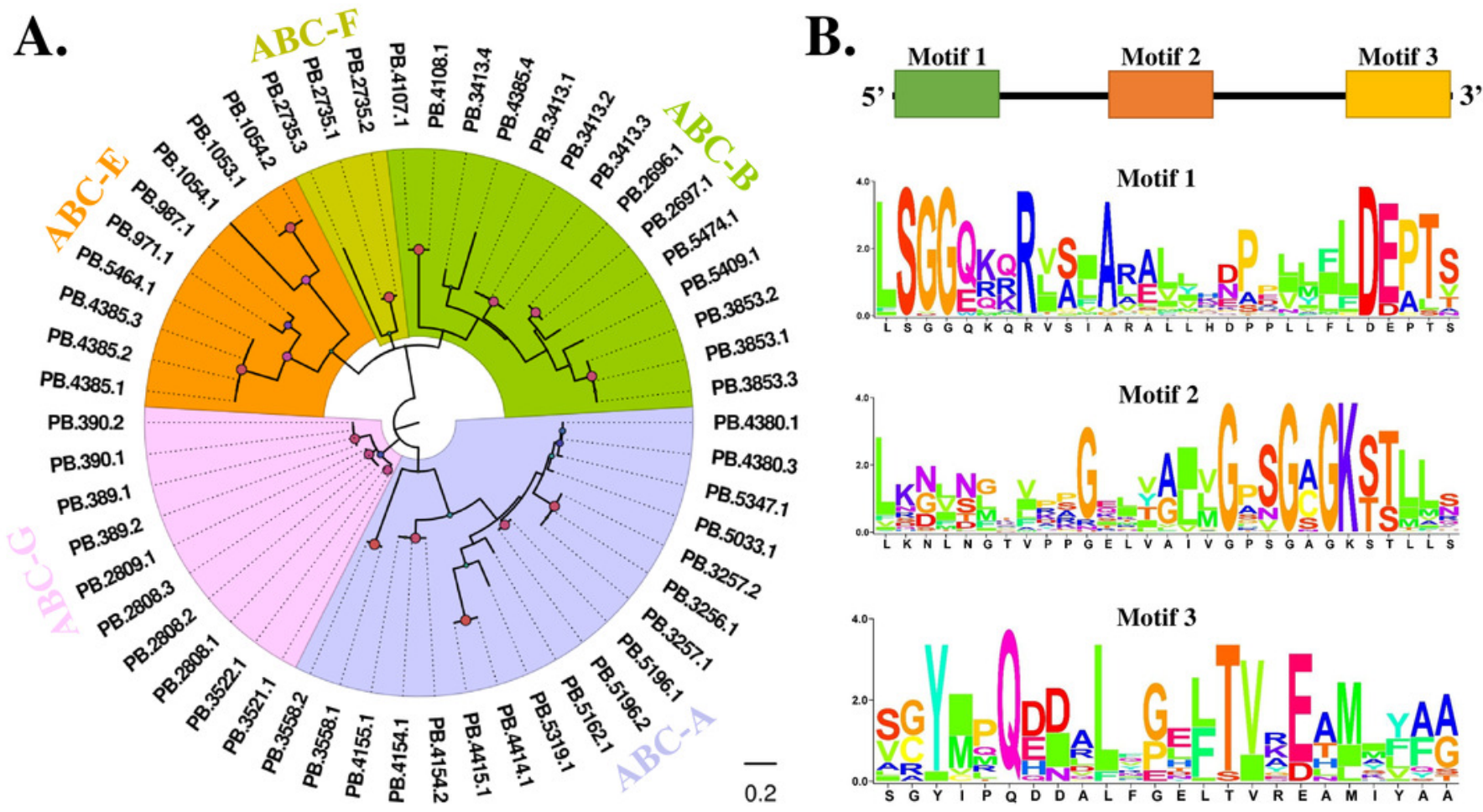
Figure 4

Figure 4: Phylogenetic and motif analysis of 46 CESs.

(A) Phylogenetic tree of 46 FAW CES transcripts. The evolutionary tree was constructed based on the maximum likelihood method using the program Mega $\mathrm{X}$ with 1000 bootstrap values. Forty-eight different amino acid substitution models were tested and "LG+G+I" was the best model. (B) Motif analysis of 46 CESs. We used MEME tool to identify the conserved motifs.
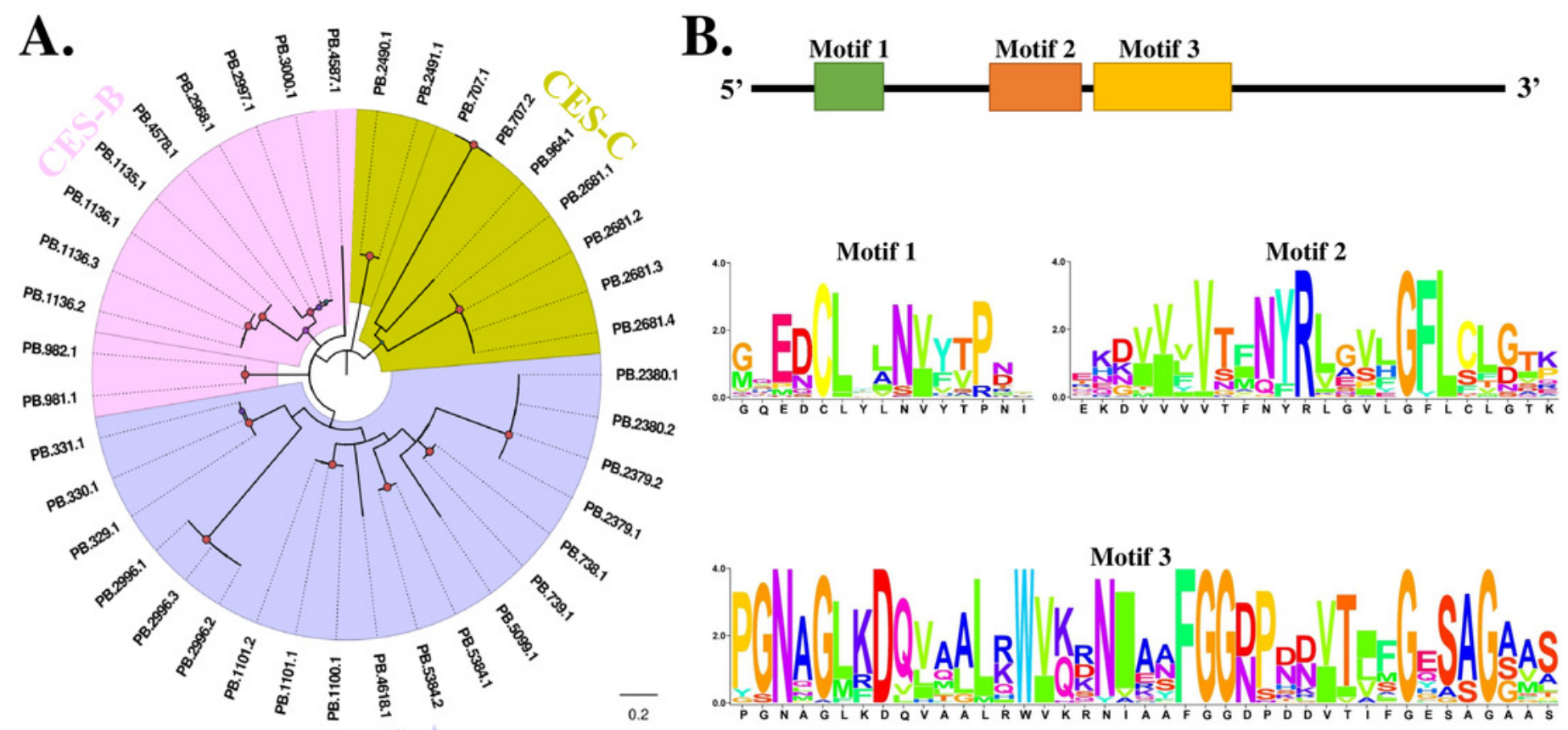


\section{Figure 5}

Figure 5: Phylogenetic construction, IncRNA target prediction and motif analysis of GSTs.

(A) Phylogenetic tree of 33 GSTs. The evolutionary tree was constructed based on maximum likelihood method using the program Mega $X$ with 1000 bootstrap values. Forty-eight different amino acid substitution models were tested and " $L G+G$ " was the best model. (B) The binding sites of IncRNA targeting transcript "PB.3383.1". LncTar was used to predict RNA targets of IncRNAs with a cutoff of -0.1 normalized deltaG. (C) Motif analysis of 33 GSTs. We used MEME tool to identify the conserved motifs. 


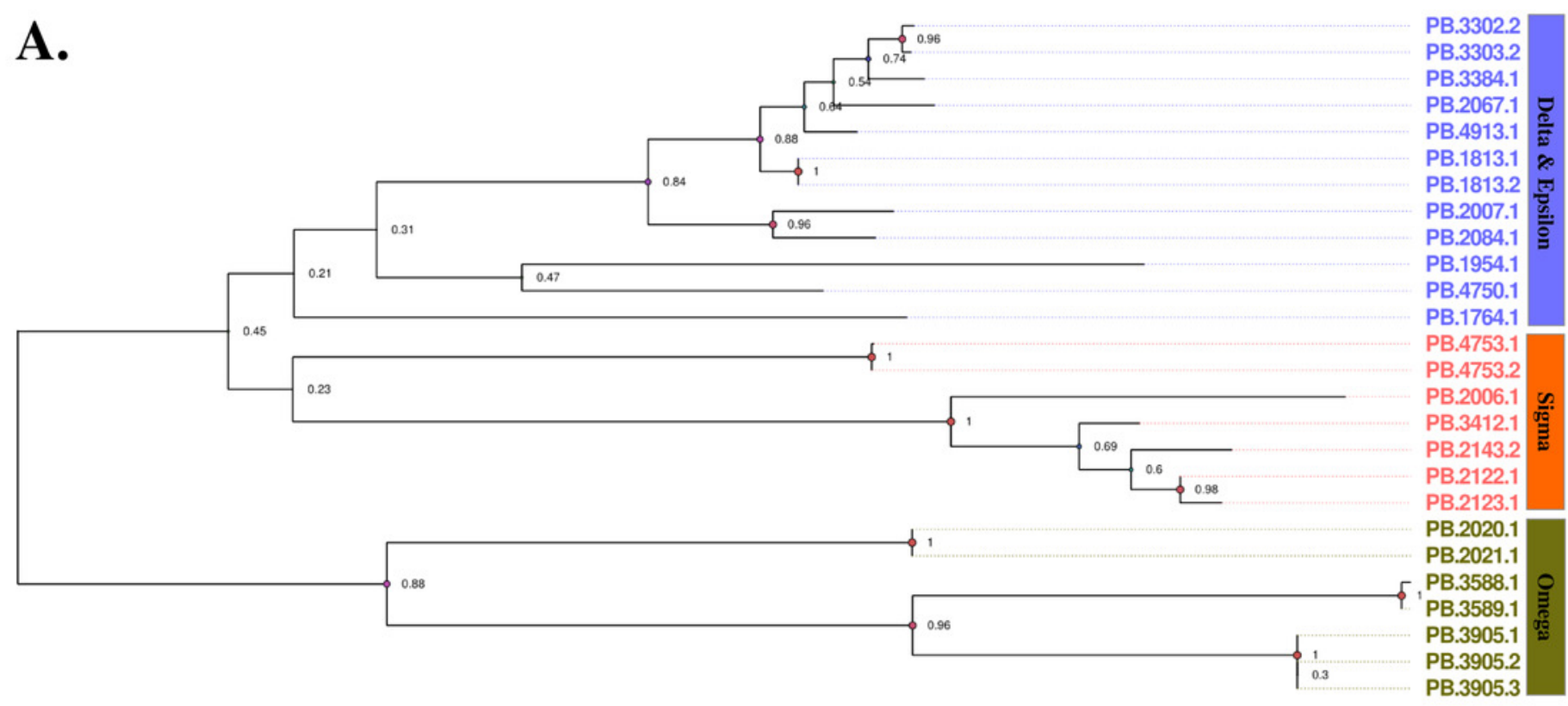

\section{B. $\quad \frac{0}{0.2}$}

Target: PB.3383.1 5' - T C T T C A AT G T A C C A T T T G A G A A G AT A A C T C T C A AT T TA C - 3’ LncRNA: transcipt 5' - AGAAGT TACATGGTAAACTC T TCT AT TGAGAGT TAAATG-3,

C.
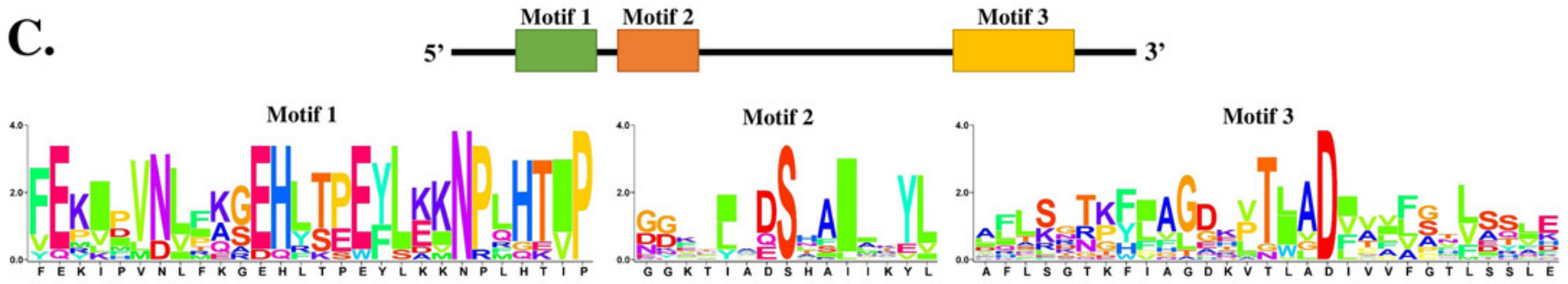
Figure 6

Figure 6: Phylogenetic and motif analysis of UGTs.

(A) Phylogenetic tree of 31 UGTs. The evolutionary tree was constructed based on maximum likelihood method using the program Mega X with 1000 bootstrap values. Forty-eight different amino acid substitution models were tested and " $\mathrm{GG}+\mathrm{G}+\mathrm{l}$ " was the best model. (B) Motif analysis of 31 UGTs. We used MEME tool to identify the conserved motifs.
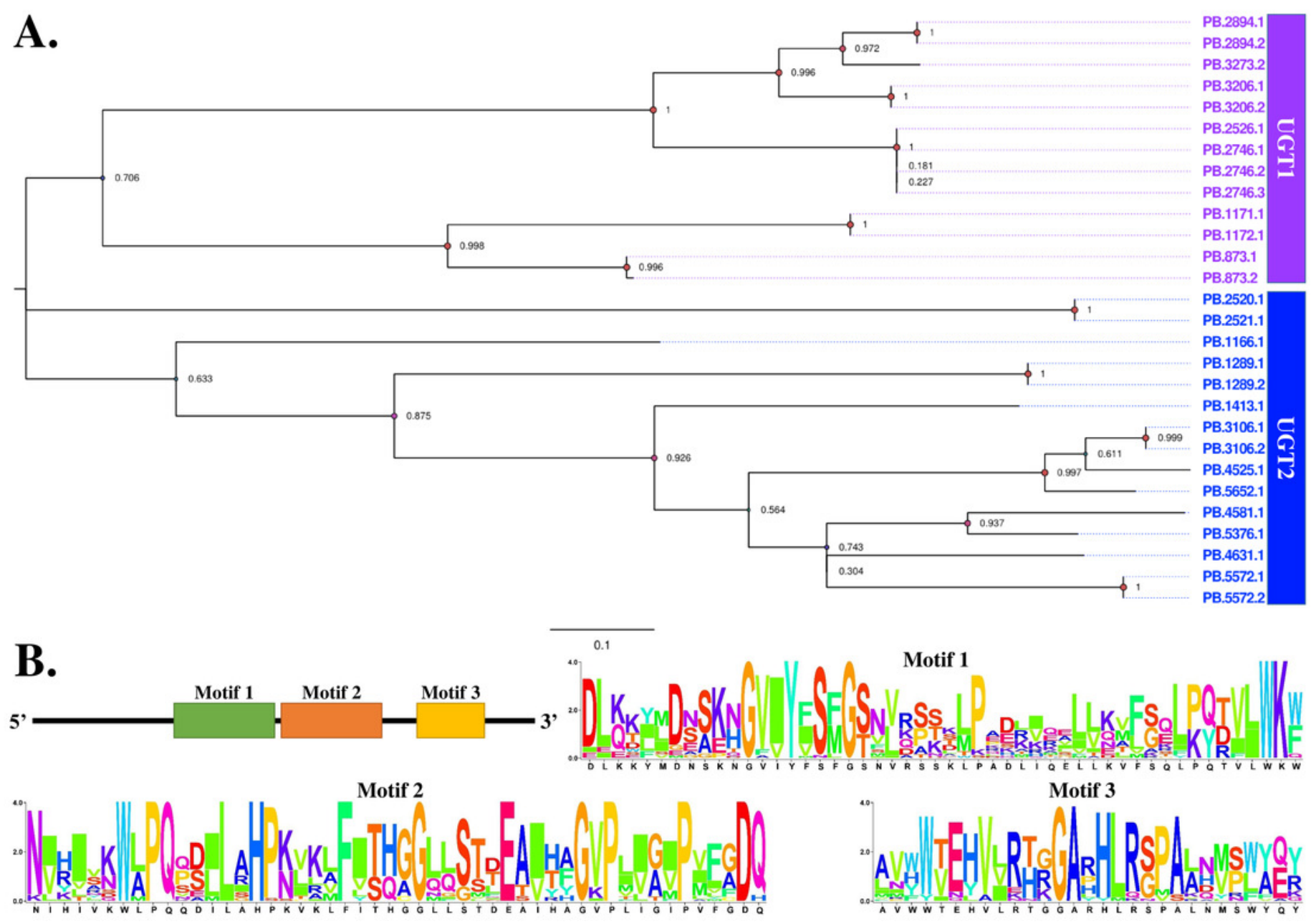